\title{
Na-Rich Interfacial Compound Impacted by Large Coherent Interfacial Strains in Na-doped p-type PbTe-PbS Themoelectrics Investigated by S/TEM
}

\author{
C. Q. Chen ${ }^{1}$, L. D. Zhao ${ }^{2}$, M. G. Kanatzidis ${ }^{2}$ and V. P. Dravid ${ }^{1}$ \\ 1. Department of Materials Science and Engineering, Northwestern University, Evanston, IL, USA \\ 2. Department of Chemistry, Northwestern University, Evanston, IL, USA.
}

The rocksalt-structured, narrow-band-gap, lead chalcogenide family of semiconductors $(\mathrm{PbX}, \mathrm{X}=\mathrm{Te}, \mathrm{S}$ and $\mathrm{Se}$ ) and their alloys continue to attract considerable scientific interest because of potential applications in thermoelectric energy conversion [1]. These lead chalcogenides semiconductors can be turned p-type by adding alkali metals as dopants. Particularly $\mathrm{Na}$, and sometimes $\mathrm{K}$, has been extensively used for this purpose. Accordingly, a precise characterization and analysis of microstructure and chemical composition particularly those associated with the distribution of $\mathrm{Na}$, as they relate to thermo-electrical transport, are vitally important for the fundamental understanding of thermoelectric materials.

$\mathrm{Na}$ dopant was conventionally considered to be homogenously distributed in the material through forming solid solutions. Recent transmission electron microscopy (TEM) and atom probe study has shown, however, that $\mathrm{Na}$ and $\mathrm{K}$ may form nanostructures in $\mathrm{PbTe}$ [2]. However, because of the extremely small size of these nanostructures it was not yet possible to determine their structure explicitly before. Here, we present our transmission electron microscopy study on $\mathrm{Na}$ doped $\mathrm{PbTe}-\mathrm{PbS}$, which is an intrinsically immiscible system with multitude of precipitates and interfaces [3]. The distribution of $\mathrm{Na}$ associated with the $\mathrm{PbTe}-\mathrm{PbS}$ interfaces are examined with analytical S/TEM.

Transmission electron microscopy results show the formation of Na-rich precipitates at the $\mathrm{PbTe}-\mathrm{PbS}$ interfaces (Fig. 1). EDS measurements clearly show an abundance of $\mathrm{Na}$ in the interfacial compound, which is composed primarily of $\mathrm{Na}$ and $\mathrm{S}$. The crystal structure of these Na-precipitates was thoroughly determined through a series of tilting experiments in TEM. (Fig. 2). The recorded set of diffraction patterns enables us to reconstruct the reciprocal lattice and to determine the lattice type and parameters. It turns out the Na-rich compound forms a long period superstructure.

Quantitative EDS analysis of the Na-rich Precipitate yields a composition approximately corresponding to $\mathrm{Na}_{3} \mathrm{~S}_{2-\mathrm{x}} \mathrm{Te}_{\mathrm{x}}$. However, it is also noted that accurate EDS quantitative analysis may need more dedicated processing of the EDS spectrum by taking into the absorption of Na-generated $\mathrm{x}$-ray by $\mathrm{S}$. The unusual structure of the new Na-rich phase is associated with large coherent interfacial stress and strains. An attempt of crystal reconstruction of the superlattice cell based on a subcell of defect anti-fluorite structure, whose lattice constants are close to the rock-salt $\mathrm{PbS}$, was performed.

The presentation will cover S/TEM microscopy and microanalysis strategy, and quantitative $\mathrm{x}$-ray analysis by considering the absorption effect, with an emphasis on unraveling the microstructural influence on thermoelectric properties and associated phenomena.

The authors acknowledge funding from ESFC. 


\section{References}

[1] Biswas, K.; He, J.; Blum, I. D.; et al. Nature, 489, 414 (2012).

[2] Girard, S. N., Klaus S. R, Thomas C. et al. Advanced Functional Materials, (2012)

[3] He, J. Q., J. Androulakis, M. G. Kanatzidis and V. P. Dravid. Nano Letters 12, 343-347, (2012):
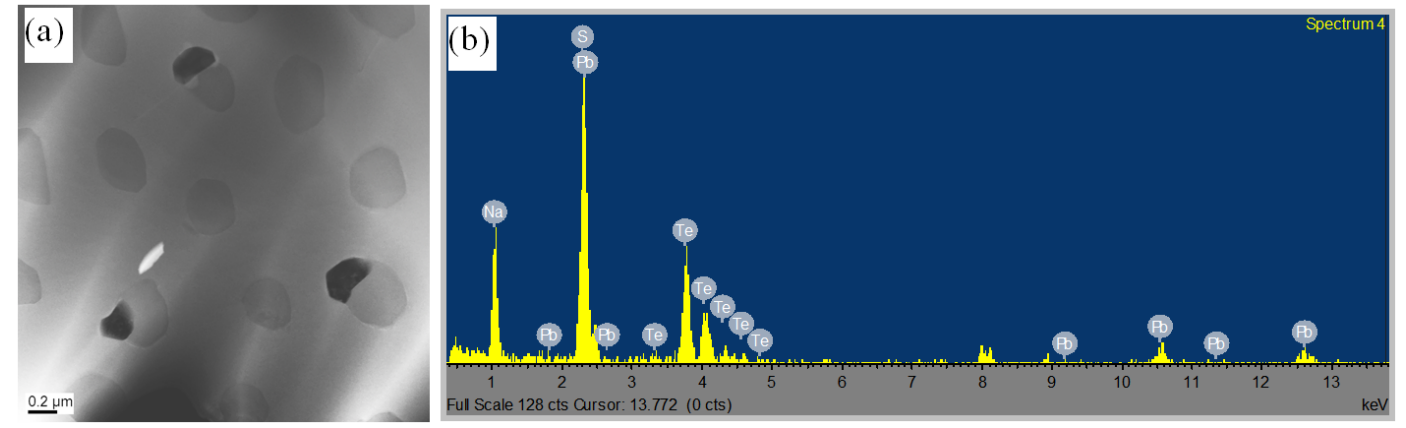

Figure 1. (a) Low magnification Z-contrast STEM image showing the structure of $\mathrm{PbTe}-\mathrm{PbS} 30 \%$ $\mathrm{Na} 2 \%$, a composition located in the spinodal decomposition regime in phase diagram. (b) EDS spectrum taken from the dark interfacial compound under STEM mode clearly showing the Na-rich nature.
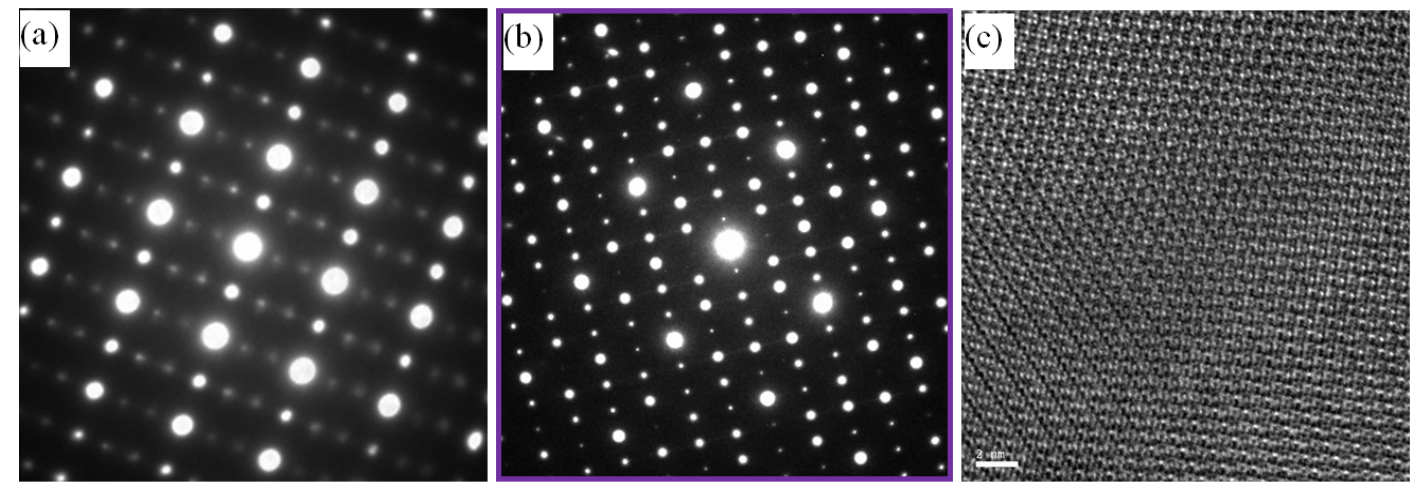

Figure 2. SAED patterns of the Na-rich compound observed in (a) [100], and (b) [001] directions. (c) HRTEM image showing clearly the modulated superstructure along $<110>$ direction. The modulated structure has a subcell that matches perfectly with the PbS rock-salt unit cell. 